\title{
Camassa-Holm equation on shallow water wave equation
}

\author{
Sh. Hajrulla ${ }^{1}$, L. Bezati ${ }^{2}$, F. Hoxha ${ }^{3}$ \\ ${ }^{1,2,3}$ University of Vlora, Albania
}

\begin{abstract}
:
In this paper we can consider the problem of week solutions for the general shallow water wave equation. In the first part of this paper, we deal to the well-known Kdv equation. We obtain the Camassa-Holm equation in particular. Both of them describe unidirectional shallow water waves equation. Moreover, all these equations have a bi-Hamiltonian structure, they are completely integrable, they have infinitely many conserved quantities. From a mathematical point of view the Camassa-Holm equation is well studied. In the second part of this paper, we obtain a global weak solution as a limit of approximation under the assumption $u_{0} \epsilon \Omega$. Some concepts related to high dimensional spaces are considered. Then the Cauchy problem is considered. It has an admissible weak solution $u=u(t, x)$ to the Cauchy problem for $u_{0} \epsilon \Omega$. Existence, uniqueness, and basic energy estimate on this approximate solution sequence are given in some lemmas. Finally, the main theorem and the proof is given.
\end{abstract}

Keywords: Kdv equation; shallow water wave equation; global solution; weak solution; Camassa-Holm equation; Cauchy problem.

\section{Introduction}

The Camassa-Holm equation and Degasperis-Procesi equation are the two equations satisfying the complete integrability condition.

For $\varepsilon=0$ in equation (1-2) we obtain the well-known Kdv equation:

$$
u_{t}+k u_{x}+\alpha u u_{x}+\gamma u_{x x x}=0
$$

In 1993 Camassa and Holm derived equation and for $\alpha=3, \quad \beta=2, \quad \gamma=0$ in equation (1-1) as a model for unidirectional water wave propagation in shallow water with u representing the height of the water's free surface above a flat bottom. The Camassa-Holm equation as follow:

$$
\left(u-\varepsilon u_{x x}\right)_{t}+k u_{x}+3 u u_{x}=\varepsilon\left(2 u_{x} u_{x x}+u u_{x x x}\right), x \in \Omega, \mathrm{t}>0
$$

Both of them describe unidirectional shallow water waves. Moreover, all these equations have a biHamiltonian structure, they are completely integrable, they have infinitely many conserved quantities. From a mathematical point of view the Camassa-Holm equation is well studied, see [11] for an extensive list of references. The relevance of the equation as a model for shallow water wave has been further investigated by Johnson. In [1]-[3], Degasperis and Procesi firstly studied the following family of third order dispersive PDE

$$
\begin{array}{ccc}
\text { conservation } & \text { laws } & \text { with } \\
u_{t}+c_{0} u_{x}+\gamma u_{x x t t} & -\alpha^{2} u_{x x}=\left(c_{1} u^{2}+c_{1} u_{x}^{2}+c_{3} u_{x x}\right)_{x}, \quad x \in \Omega, \mathrm{t}>0
\end{array}
$$
conditions.

where $\alpha, c_{0}, c_{1}, c_{2}, c_{3}$ are real constant and indices denote partial derivatives. The notation, $\Omega$ is a domain where the functions are defined, for example $\Omega=[0,1]$.

Following the methodology described in [6] for general bi-Hamiltonian systems, it is possible to derive an infinite number of conserved quantities for the solutions of (1-1). The problem consists of computing the scattered far-field over an obstacle whose "shape" is determined in some way by $u$ (for $t$-fixed). In 
practice, it means finding the eigenvalues of a linear operator depending on $u(t, x)$. The remarkable fact is that as time evolves, if $u(t, x)$ satisfies (1-1), then these eigenvalues satisfy trivial linear ordinary differential equations which can be solved explicitly and the far-field can be determined for any time. The inverse scattering problem consists of retrieving the "shape" of the obstacle, that is $u$, from the knowledge of the scattered far-field.

When $c_{1}=\frac{\alpha}{2}$ and $c_{2}=\frac{\varepsilon(\beta-1)}{2}$ and $c_{3}=\varepsilon$, than replacing $c_{0}$ with $k$ and $\alpha^{2}$ with $\varepsilon$ in the equation (1-1), we obtain the general shallow water equation as follow:

$$
\left(u-\varepsilon u_{x x}\right)+k u_{x}+\alpha u u_{x}+\gamma u_{x x x}=\varepsilon\left(\beta u_{x} u_{x x}+u u_{x x x}\right), x \in \Omega, \mathrm{t}>0
$$

There are at least three famous equations that satisfy the completely integrability condition within this family: KdV equation (see [4]), Camassa-Holm equation (see [5][16]), and Degasperis-Procesi equation (see [1]-[3]).

The shallow water equations model the propagation of disturbances in water and other incompressible fluids. Shallow water flows are characterized by flow regions with a free surface, an impermeable The shallow water wave equations form a non-linear hyperbolic system. The equations often admit discontinuous solutions even when the initial data is smooth and analytical solutions are limited to very few idealized cases.

The depth of the fluid is small compared to the wave length of the disturbance. For example, we do not ordinary think of the Indian Ocean as being shallow. The depth is two or three kilometers. But the devastating tsunami in the Indian Ocean on December 26, 2004 involved waves that were dozens or hundred of kilometers long. So the shallow water approximation provides a reasonable model in this situation.

In particular, we recall that existence and uniqueness results for global weak solutions have been proved by Coclite et al. [11], Constantin and Escher [12], and Xin and Zhang [13].

In Section 2, we give some knowledges related to $\mathrm{KdV}$ equation, than give the statements of the main results. Section 4 deals with the viscous approximate solutions and the basic energy estimate on $u_{0}$. Some explanation related to the conservation law of energy is treated.

\section{Preliminary}

The Korteweg-deVries equation (the KdV equation), is perhaps the simplest nonlinear partial differential equation: $\quad u_{t}+6 u u_{x}+u_{x x x}=0$

where $u=u(x, t)$ is a function of two variables. The $\mathrm{KdV}$ equation is extremely important as it arises in many physical contexts. It can be used to describe waves in shallow water, anharmonic nonlinear lattices, gas dynamics, and hydromagnetic and ion-acoustic waves in cold plasma, for example. The interested reader is for the physical derivations. Note that the coefficients in front of the three terms are somewhat arbitrary.

The Modified KdV Equation. A similar equation that will play an important role in what follows is known as the Modified KdV equation, abbreviated the MKdV equation:

$$
v_{t}+6 v^{2} v_{x}+v_{x x x}=0
$$

In the course of attempting to solve the $\mathrm{KdV}$ equation exactly, it was discovered that the equation has an infinite sequence of nontrivial conservation laws, which we shall presently define.

\section{Some notes:}

\section{Functional space of $\boldsymbol{C}^{\boldsymbol{m}}(\Omega)$}

Def: Functional space is a set of functions with operations.

Example: $C(\Omega)=C^{m}(\Omega)=\{\mathrm{u}(\mathrm{x}), \mathrm{u}(\mathrm{x})$ is a continuous on $\Omega\}$ is a linear space that contains all continuous functions on $\Omega$. $C^{m}(\Omega)=\left\{u(x), u(x), u^{\prime}(x), \ldots, u^{m}\right.$ are continuous on $\left.\Omega\right\}$.

We define: $C^{\infty}(\Omega)=\{u(x), \quad u(x)$ isindefinitely differentiable on $\Omega\}$.

\section{Sobolev spaces $H^{m}(\Omega)$}

Recall the $C^{m}(\Omega)$ spaces. For analogy define the integral spaces $H^{m}(\Omega)$.

Def. The integral spaces $H^{m}(\Omega)$ is called, $C^{0} \rightarrow L^{2}=H^{0}, \quad C^{m} \rightarrow L^{m}$ 
The square-integrable space $H^{0}(\Omega)=L^{2}(\Omega)$ is $L^{2}(\Omega)=\left\{u(x) \int_{\Omega} u^{2}(x) d(x)<\infty\right\}$

Claim: $L^{2}(\Omega)$ is a complete space. This means that any Cauchy sequence $\left\{f_{n}\right\}$ in $L^{2}$. In other words, there is a $f \in L^{2}(\Omega)$ such that $\lim _{n \rightarrow \infty}\left\|f_{n}-f\right\|_{L^{2}}=0$ or $\lim _{n \rightarrow \infty} f_{n}=f$

Def. A Cauchy sequence is a sequence that satisfies the property: for any given positive number $\varepsilon$, there is an integer $N$, such that $\left\|f_{n}-f\right\|_{L^{2}}<\varepsilon$, if $m \geq N, \quad n \geq N$.

Def. A complete normed space is called a Banach space (a Cauchy sequence converges in terms of the norm). Therefore $L^{2}(\Omega$ is a Banach space.

\section{Definition of Sobolev spaces $\boldsymbol{H}^{m}(\Omega)$}

Having defined the first partial derivatives, we define the Sobolev spaces $H^{1}(\Omega)$ as

$$
H^{1}(\Omega)=\left\{v(x), \quad D^{|\alpha|} v \in L^{2}(\Omega), \quad|\alpha| \leq 1\right\}
$$

Def. We denote the norm in Sobolev space $H^{m}(\Omega)$ as

$\left.\|u\|_{p}=\|u\|_{H}=\left\{\int_{\Omega}\left(1+|v|^{2}\right)+|f(v)|^{2}\right) d v\right\}^{1 / 2}$, where $f(v)$ is the Fourier transform of $f(x)$.

\section{Sobolev spaces-related to derivatives in integral forms}

Similar to $C^{m}(\Omega)$, we use $H^{m}(\Omega)$ to define function spaces with derivatives in integral forms. Such functions spaces are part of Sobolev spaces.

If there is no derivative, we define: $H^{0}(\Omega)=L^{2}(\Omega)=\left\{v(x), \int_{\Omega}\left|v^{2}\right| d x<\infty\right\}$

The $L^{p}(\Omega)$ spaces

Def . The $L^{p}(\Omega)$ spaces is defined as

Def. The distance in $L^{p}(\Omega)$ is defined as $\quad d(f, g)=\left\{\int_{\Omega}|f-g|^{p} d(x)\right\}^{\frac{1}{p}}$

Notation: We shall use the standard notation $|.|_{p}$ for the norm of the space $L^{p}(\Omega), 1 \leq p<\infty$ (i.e. $|\boldsymbol{f}|_{\boldsymbol{p}}=\left\{\int_{\Omega}|f|^{p} d(x)\right\}^{\frac{1}{p}}$ )

\section{Global weak solution and the main theorem}

Before giving the precise statements of the main results, we introduce the definition of a weak solution to the Cauchy problem ( 1-2).

Def 1. A continuous function $\mathrm{u}=\mathrm{u}(t, x)$ is a global weak solution to the Cauchy problem

(1.2) if: $\mathrm{u}=\mathrm{u}(t, x) \in C((0, \infty) \times \Omega) \cap L^{\infty}\left(R, H^{m}(\Omega)\right)$ and $\|u\|_{H^{m}(\Omega)} \leq\left\|u_{0}\right\|_{H^{m}(\Omega)} \quad \forall t>0$ $\mathrm{u}(t, x)$ satisfies equation (1-2) in the sense of distributions.

Note: $\Omega \subset R^{n}, \mathrm{n} \geq 1$, is a bounded domain with smooth boundary $\partial \Omega$.

Theorem 2. Suppose that $u_{0} \in H^{m}(\Omega)$. Then the Cauchy problem (1.2) has an admissible weak solution $\mathrm{u}=\mathrm{u}(t, x)$ in the sense of Def 1 above. Furthermore, the weak solution $\mathrm{u}(t, x)$ to the Cauchy problem ( 1 -

2) satisfies the following properties:

1) (Oleinik type estimate) There exists a positive constant $C$ depending only on $\left\|u_{0}\right\|_{H^{m}(\Omega)}$ such that

$$
\partial_{x} u(t, x) \leq \frac{1}{t}+C \text { for all } t>0
$$

2) $P(t, x) \in L^{\infty}\left(R^{+}, H^{m}(\Omega)\right)$ and $\partial_{x} u(t, x) \in L^{p}\left(R^{+}, H^{m}(\Omega)\right)$ for any $p<3$ (i.e. for any $0<T, M<$ $+\infty$, there exist a positive constant $C_{1}=C_{1}(T, M, p)$ such that

$$
\int_{0}^{T} \int_{|x| \leq 3}\left|\partial_{x} u(t, x)\right|^{p} d x d t \leq C_{1} \text { for all } p<3
$$


3) Assume $u(t, x)$ is of one sign, than $\mathrm{u}=\mathrm{u}(t, x)$ approaches zero point wise as $t \rightarrow \infty$, i.e. $\lim _{t \rightarrow \infty}|u(t, x)|=0$, for all $x \in \Omega\left(\Omega \subset R^{n}, \mathrm{n} \geq 1\right)$

\section{Some lemmas and proof of the Theorem 2}

In this section, supposing $\varepsilon=1$ in Eq. (1-2), we construct the approximate solution sequence $u_{\varepsilon}=u_{\varepsilon}(t, x)$ as solutions to the Cauchy problem (4-1), i.e.

$$
\left\{\begin{array}{c}
\partial_{t} u_{\varepsilon}+u_{\varepsilon} \partial_{x} u_{\varepsilon}-\gamma \partial_{x} u_{\varepsilon}+\partial_{x} P_{\varepsilon}=\mu \partial_{x}{ }^{2} u_{\varepsilon} \\
P_{\varepsilon}=\frac{1}{2} \int_{-\infty}^{+\infty} e^{-|x-y|}\left(\frac{\alpha-1}{2} u_{\varepsilon}{ }^{2}+\frac{3-\beta}{2}\left(\partial_{x} u_{\varepsilon)}{ }^{2}+(k+\gamma) u_{\varepsilon}\right)(t, y) d y\right. \\
u_{\varepsilon}(0, x)=u_{\varepsilon_{0}}(t, x)
\end{array}\right.
$$

The existence, uniqueness, and basic energy estimate on this approximate solution sequence are given in the following lemma:

Lemma 3(Propos. 3): Let $\varepsilon>0$ and $u_{\varepsilon_{0}} \in H^{k}(\Omega)$ for some $\mathrm{k} \geq 2$. Then there exist a unique solution $u_{\varepsilon}=u_{\varepsilon}(t, x) \in C\left((0, \infty), H^{k}(\Omega)\right)$ to the Cauchy problem (3.1). Furthermore, $u_{\varepsilon}$ satisfies

$$
\left\|u_{\varepsilon}\right\|_{H^{m}(\Omega)} \leq\left\|u_{\varepsilon_{0}}\right\|_{H^{m}(\Omega)} \quad \forall t>0
$$

Lemma 4(Propos. 4): Let $u_{0} \in H^{m}(\Omega)$ and $u_{\varepsilon}(t, x)$ be the solution to the Cauchy problem (4-1). There exist a positive constant $C$ depending only on $\left\|u_{0}\right\|_{H^{m}(\Omega)}$, such that

$$
\partial_{t} u_{\varepsilon}(t, x) \leq \frac{1}{t}+C \text { for all } t>0, x \in R
$$

Lemma 5(Propos. 5): Let $m=\frac{2 k}{2 k+1}$ with $l . k$ being positive integers and $l \geq k$. Assume that $a, b, T$ are arbitrarily given finite constants with $a<b$ and $T>0$. Then there exists a positive constant $C=$ $C\left(a, b, T, m,\left\|u_{0}\right\|_{H^{m}(\Omega)}\right)$ independent of $\varepsilon$, such that

$$
\int_{0}^{T} \int_{a}^{b}\left|\partial_{t} u_{\varepsilon}(t, x)\right|^{2+m} d x d t \leq C
$$

We start with the weak compactness in $L^{\infty}\left(R^{+}, H^{m}(\Omega)\right)$. We are now ready to obtain the necessary compactness of the viscous approximate solution $u_{\varepsilon}(t, x$.

Lemma 6(Propos. 6): There exist a subsequence $\left\{u_{\varepsilon_{j}}(t, x), P_{\varepsilon_{j}}(t, x)\right\}$ of the sequence $\left\{u_{\varepsilon}(t, x), P_{\varepsilon}(t, x)\right\}$ and some functions $\{u(t, x), P(t, x)\}, u \in L^{\infty}\left(R^{+}, H^{m}(\Omega)\right)$ and $P \in L^{\infty}\left(R^{+}, H^{m}(\Omega)\right)$, such that $u_{\varepsilon_{j}} \rightarrow u$ as $j \rightarrow \infty$ and $P_{\varepsilon_{j}} \rightarrow P$ in $L^{q}\left(R^{+}, H^{m}(\Omega)\right)$ as $j \rightarrow \infty$ for all $1<q<+\infty$.

Next, we show the stronger result, i.e. $\partial_{t} u_{\varepsilon}(t, x) \rightarrow \partial_{t} u(t, x)$ in $L^{2}\left(R^{+}, H^{m}(\Omega)\right)$ as $\varepsilon \rightarrow 0^{+}$, which will guarantee that $u(t, x)$ is a desired weak solution. First, we state a slight variation of the basic result of the theory of Young measures ( see [16 - 18]).

Lemma 7(Propos. 7): Let $u_{t, x}(\lambda)$ be the Yang measure associated with $\left\{q_{\varepsilon}(t, x)\right\}=\left\{\partial_{x} u_{\varepsilon}(t, x)\right\}$.

Than for any continuous function $f=f(\lambda)$ with $f(\lambda)=o\left(|\lambda|^{r}\right)$ and $\partial_{\lambda} f(\lambda)=o\left(|\lambda|^{r-1}\right)$ as $|\lambda| \rightarrow \infty$ and $r<2$ and for any $\psi \in L_{c}{ }^{s}(\Omega)$ with $\frac{1}{s}+\frac{r}{2}=1$ we have 


$$
\lim _{\varepsilon \rightarrow 0} \int_{\Omega} f\left(q_{\varepsilon}(t, x)\right) \psi(\mathrm{x}) d x=\int_{\Omega} \overline{f(q)} \psi(\mathrm{x}) d x
$$

uniformly in each compact subset $\Omega$, where

$$
\overline{f(q)}=\int_{\Omega} f(\lambda) d \eta_{t, x}(\lambda)
$$

Lemma 8(Propos. 8): Let $u_{t, x}(\lambda)$ be the young measure given in Lemma 7 . Then

$$
u_{t, x}(\lambda)=\delta_{\bar{q}(t, x)}(\lambda) \quad \text { for almost all }(t, x) \in R_{+} \times \Omega
$$

\section{Proof of the Theorem}

With all the preparation given in the previous section, we are now in a position to prove the main results i.e., Theorem 2. Let $(u, P)(t, x)$ be the limit of the viscous approximate solutions $\left(u_{\epsilon}, P_{\epsilon}\right)(t, x)$ as $\epsilon \rightarrow 0^{+}$. It then follows from Lemma 3, 4 and 6 that $u \in C([0, \infty) \times R) \cap L^{\infty}\left(R^{+}, H^{m}(\Omega), \quad P \in L^{\infty}\left(R^{+}, W^{1, \infty}(\Omega)\right.\right.$. Taking $\epsilon \rightarrow 0^{+}$in $(3.1)$, on can see free the Proposition 6 that $(u, P)(t, x)$ will be an admissible weak solution provided that

$$
q_{\epsilon} \equiv \partial_{x} u_{\epsilon} \rightarrow q=\partial_{x} u \quad \text { in } \quad L_{l o c}^{2}\left(R_{+} \times \Omega\right)
$$

as $\epsilon \rightarrow 0^{+}$. However (5.1) is now a simple consequence of Lemma 8 and Lemma 7. In fact it follows (4.6)-(4.7) and Lemma 8 that there exists a subsequence of $\left\{u_{\epsilon}(t, x)\right\}$, still denoted by itself, such that $q_{\epsilon} \equiv \partial_{x} u_{\epsilon} \rightarrow q=\partial_{x} u \quad$ in $\quad L_{l o c}^{p_{1}}\left(R_{+}, L_{l o c}^{p_{2}}(\Omega)\right) \forall p_{1}<\infty, p_{2}<2$

This together with Lemma 5 implies $\quad q_{\epsilon} \equiv \partial_{x} u_{\epsilon} \rightarrow q=\partial_{x} u \quad$ in $\quad L_{l o c}^{p}\left(R_{+} \times \Omega\right) \forall p<3$

by a simple interpolation, which gives (5.1) immediately. Furthermore, it follows from (5.3) that

$$
\partial_{x} u \in L_{l o c}^{p}\left(R_{+} \times \Omega\right) \forall p<3
$$

hence the local space-time higher integrability estimate, holds.

Last step of the proof, is to investigate the asymptotic behavior of the solution $u(t, x)$. By our assumption that $u(t, x)$ is one of the sign, we will consider the nonnegative solution only. The other case is similar. Let $u(t, x) \geq 0$ be an admissible weak solution to equation (1.2) with $\epsilon=1$. Then for any $t \in R_{+}$, integrate equation $(1.2)$ over $[0, t] \times(-\infty, x]$ to get

$$
\int_{-\infty}^{x} u(t, y) d y+\frac{1}{2} \int_{0}^{t} u^{2}(s, x) d s-\gamma \int_{0}^{t} u(s, x) d s+\int_{0}^{t} P(s, x) d s=\int_{-\infty}^{x} u_{0}(y) d y
$$

We have: $\|u(t,)\|_{L^{1} R}+\frac{1}{2}\|u(, x)\|_{L^{2}\left(R_{+}\right)}-\gamma\|u(, x)\|_{L^{1}\left(R_{+}\right)}+\|P(, x)\|_{L^{1}\left(R_{+}\right)} \leq\left\|u_{0}\right\|_{L^{1}(R)}$

On the other hand, from the standard estimate on convolution one can get

$$
\left\|\partial_{x} P(t,)\right\|_{L^{2}} \leq C_{0}\left\|u_{0}\right\|_{H^{1}}^{2}, \text { which shows that } \quad \partial_{x} u \in L^{\infty}\left(R_{+}, L^{2}(\Omega)\right.
$$

Hence, there exists a subset $N \in R$ with meas $(N)=0$ such that $\partial_{x} u \in L^{\infty}\left(R_{+}\right)$for all $x \in R \backslash N$. It follows from this and (5.5) that $\quad \lim _{t \rightarrow+\infty}|u(t, x)|=0 \quad$ for $x \in R \backslash N$ 
For any $x \in N$, there is a sequence $\left\{x_{j}\right\} \in R \backslash N$, such that $x_{j} \rightarrow x$ as $j \rightarrow+\infty$. Since

$$
|u(t, x)| \leq \mid u\left(t, x_{j}|+| u(t, x)-u\left(t, x_{j}|\leq| u\left(t, x_{j}\left|+C_{0}\right| x-x_{j} \mid\right.\right.\right.
$$

we conclude that

$$
\lim _{t \rightarrow+\infty}|u(t, x)|=0 \quad \text { for } \quad x \in N
$$

Then (2.4) follows from (5.6) and (5.7). This complete the proof of the Theorem.

\section{Conclusion}

Such wave breaking is observed in fluid flows, and under this aspect, the Camassa-Holm equation could be seen as a more suitable model than the well known Korteweg-de Vries equation, for instance. On the other hand, in the derivation of the Camassa-Holm equation, it is assumed that the solutions are more regular than breaking waves [19]. In this respect, smooth solutions are more closely related to the fluid flow problem than are irregular solutions. From this point of view, a spectral approximation seems a natural choice for a spatial discretization. Another application of the Camassa-Holm equation arises when $\gamma=0$. In this case, the equation can be derived as a model equation for mechanical vibrations in a compressible elastic rod.

Notwithstanding its importance as a model equation, one reason for the interest in the Camassa-Holm equation is its vast supply of novel mathematical issues, such as its integrable bi-Hamiltonian structure. This property alone has led to many interesting developments. One aspect of the integrability of the equation in case $\gamma=1$ is that the solitary-wave solutions are solitons, similar to the solitary-wave solutions of the Korteweg-de Vries equation.

However, the Camassa-Holm equation also admits solitary waves which are not smooth, but rather have a peak or even a cusp. These peaked solitary waves are well known, and owing to their soliton-like properties they have been termed peakons.

For the purpose of numerical study, it is important to have a satisfactory theory of existence of solutions, as well as uniqueness and continuous dependence with respect to the initial data.

\section{References}

1. L. Bona, R. Scott: Solutions of the Korteweg-de Vries equation in fractional order Sobolev spaces.DukeMath.J..43:67-79(1976).

2. Constantin A., Escher J.: Global weak solutions for a shallow water equation. Indiana Univ. Math. $J . .47: 1500-1511(1998)$

3. A. Degasperis, D. Holm, A. N.W. Hone: Integrable and non-integrable equations with peakons. in:Nonlinear Physics: Theory and Experiment, vol. II, Gallipoli,2002. World Scientific, River Edge, NJ. (2003)

4. J.L. Bona, R. Smith: The initial-value problem for the Korteweg-de Vries equation. Philos. Trans. R. Soc. Lond. . 278: 555-601(1975) IJNS homepage:http://www.nonlinearscience.org.uk/

5. R. Camassa, D. Holm: An integrable shallow water equation with peaked solutions. hys. Rev. Lett. 71: 1661-1664(1993)

6. J. R. S. Johnson. Camassa\{Holm, Korteweg-de Vries and related models for water waves. J. Fluid Mech., 455:63\{82, 2002

7. Ping Zhang: Weak solutions to a nonlinear variational wave equation and some related problems. Applications of Mathematics. 4: 287-306(2006)

8. Lixin Tian, Guilong Gui, Yue Liu: On the Cauchy problem for the generalized shallow water wave equation. J. Diff. Equ.. 265(7): 1838-1852(2008)

9. Guilong Gui, Yue Liu, Lixin Tian: Global existence and blow-up phenomena for the peakon bfamily of equations. Indiana Univ. Math. J.. 57(3): 1209-1234(2008)

10. Jiangbo Zhou, Lixin Tian: A type of bounded traveling wave solutions for the Fornberg-Whitham equation. J. Math. Anal. Appl.. 346: 255-261(2008)

11. Coclite G.M., Holden H., Karlsen K.H.: Global weak solutions to a generalized hyperelastic-rod wave equation. SIAM J. Math. Anal.. 37: 1044-1069(2005) 
12. Constantin A., Escher J.: Global weak solutions for a shallow water equation. Indiana Univ. Math. J.. 47: 1527-1545(1998)

13. Constantin A., Escher J.: Global weak solutions for a shallow water equation. Indiana Univ. Math. J.. 47: 1527-1545(1998)

14. Zhouping Xin, Ping Zhang: On the weak solutions to a shallow water equation Commun. Pure Appl. Math.. 53: 1411-1433(2000)

15. Danchin R.: A note on well-posedness for Camassa-Holm equation. J. Diff.qu.192 in: Lecture Notes in Math., 448: 25-70(1975) 429-444(2003) W.N. Everitt (Ed.). Differential Equations

16. Ping Zhang: Weak solutions to a nonlinear variational wave equation and some related problems. Applications of Mathematics. 4: 427-466(2006)

17. Ping Zhang, Yuxi Zheng: Existence and uniqueness of solutions to an asymptotic equation arising from a variational wave equation with general data. Arch. Ration Anal. . 155: 49-83(2000)

18. Constantin A., Escher J.: Global weak solutions for a shallow water equation. Indiana Univ. Math. J.. 47: 1507-1515(1998)

19. H. Holden and $X$. Raynaud. A convergent numerical scheme for the camassa\{holm equation based on multipeakons. Discrete Contin. Dyn. Syst., 14(3), 2006 